Bruno do Carmo Pontes

\title{
Simulação Estática e Dinâmica do Controle de Tensão por LTC e Compensador Estático
}

Dissertação de Mestrado

Dissertação apresentada como requisito parcial para obtenção do título de Mestre pelo Programa de PósGraduação em Engenharia Elétrica da PUC-Rio.

Orientador: Ricardo Bernardo Prada Co-orientador: José Eduardo Onoda Pessanha

Rio de Janeiro

Agosto de 2008 


\section{Bruno do Carmo Pontes}

\section{Simulação Estática e Dinâmica do Controle de Tensão por Gerador e Compensador Síncrono}

Dissertação apresentada como requisito parcial para obtenção do grau de Mestre pelo Programa de Pós-Graduação em Engenharia Elétrica do Departamento de Engenharia Elétrica do Centro Técnico Científico da PUC-Rio. Aprovada pela Comissão Examinadora abaixo assinada.

Prof. Ricardo Bernardo Prada

Orientador

Departamento de Engenharia Elétrica/PUC-Rio

Prof. José Eduardo Onoda Pessanha

Co-Orientador

UFMA

Prof. Ricardo Diniz Rangel

CEPEL

Prof. Glauco Nery Taranto

COPPE/UFRJ

Prof. José Eugenio Leal Coordenador Setorial do Centro

Técnico Cinetífico - PUC-Rio

Rio de Janeiro, 11 de agosto de 2008 
Todos os direitos reservados. É proibida a reprodução total ou parcial do trabalho sem autorização da universidade, do autor e do orientador.

\section{Bruno do Carmo Pontes}

Técnico em Eletrotécnica pelo CEFET-RJ e graduado Engenheiro Eletricista pela UERJ em 2004. De 1999 a junho de 2006 trabalhou no Centro de Operação do Sistema Elétrico de Furnas Centrais Elétricas S.A. como técnico, na operação do sistema elétrico, nas atividades de tempo real. Em julho de 2006 foi aprovado como Engenheiro Eletricista em concurso público para a Eletrobrás Centrais Elétricas S.A.. Desde então trabalha no Departamento de Comercialização da empresa, assumindo a atividade de operação e comercialização da UHE Itaipu, do Proinfa - Programa de Incentivo às Fontes Alternativas de Energia e ainda das conversoras internacionais de Rivera (Uruguai) e Uruguaiana (Argentina).

Ficha catalográfica

Pontes, Bruno do Carmo

Simulação estática e dinâmica do controle de tensão por LTC e compensador estático / Bruno do Carmo Pontes ; orientador: Ricardo Bernardo Prada ; co-orientador: José Eduardo Onoda Pessanha. - 2008.

194 f. : il. (color.) ; $30 \mathrm{~cm}$

Dissertação (Mestrado em Engenharia Elétrica)-Pontifícia Universidade Católica do Rio de Janeiro, Rio de Janeiro, 2008.

Inclui bibliografia

1. Engenharia elétrica - Teses. 2. Estabilidade de tensão. 3. Instabilidade de tensão 4. LTC. 5. Transformadores. 6. Simulação estática. 7. Simulação dinâmica. 8. Compensador estático de reativos. I. Prada, Ricardo Bernardo. II. Pessanha, José Eduardo Onoda. III. Pontifícia Universidade Católica do Rio de Janeiro. Departamento de Engenharia Elétrica. IV. Título. 
Este trabalho é dedicado a memória de Diogo do Carmo Pontes por ter me ensinado a nunca desistir, por maior que seja a dificuldade.

Sua lembrança nos remete à verdadeira alegria de viver. Em 2008 faz 10 anos que ele nos deixou. 


\section{Agradecimentos}

Ao Prof. Ricardo Prada pela orientação.

Ao Prof. José Pessanha e aos doutorandos Alex Ricardo Arquiñego Paz e Carlos Enrique Portugal Poma, todos da UFMA, pela colaboração nas horas de dificuldade.

Ao CEPEL - Centro de Pesquisas de Energia Elétrica, empresa do Grupo Eletrobras, pela cessão dos programas ANAREDE e ANATEM, utilizados nesta dissertação.

Ao também mestrando Armando Gonçalves Leite, da PUC-Rio, pela bem sucedida parceria de 13 anos (CEFET-RJ, UERJ, PUC-Rio, Furnas e Eletrobrás).

À minha mãe Sonia, por tudo que ela sempre foi, pelo que ela é e pelo que ela sempre representará para mim.

Aos meus pais, pais "emprestados" e minha família, pelo apoio.

À minha namorada Sissi, por ter sido compreensiva e me apoiado nas horas difíceis.

Aos amigos do CTB LIII, de Furnas, pela cumplicidade, pelas trocas, pela ajuda e principalmente pela amizade.

Aos meus amigos, pelo apoio, pela compreensão nesse tempo que estive "ausente".

À vida.

A Deus. 


\section{Resumo}

Pontes, Bruno do Carmo; Prada, Ricardo Bernardo. Simulação Estática e Dinâmica do Controle de Tensão por LTC e Compensador Estático . Rio de Janeiro, 2008. 194 p. Dissertação de Mestrado - Departamento de Engenharia Elétrica, Pontifícia Universidade Católica do Rio de Janeiro.

O tema abordado neste trabalho é a observação e análise, em regime permanente e dinâmico, da ocorrência de um fenômeno que já foi observado em condições reais de operação do sistema elétrico brasileiro, que é a relação oposta à usual entre a grandeza controlada e a grandeza controladora. Nestes caso, mesmo que haja margem de recursos para manter a tensão controlada, ela não é útil. Por exemplo, uma diminuição na relação de transformação num transformador de tapes variáveis, com intuito de aumentar a tensão controlada acaba por reduzí-la, até que os limites de troca de tapes sejam atingidos ou o sistema entre em colapso. Para demonstrar a existência do problema, foram executadas simulações, em regime permanente e dinâmico, e verificado o efeito do controle de tensão por um transformador com tapes variáveis e por compensadores estáticos de potência reativa, situações corriqueiras de um sistema de potência. Foram demonstradas situações em que foi possível verificar a mudança da região de operação. Para a análise em regime permanente foi utilizado um algoritmo de fluxo de carga, e para a análise dinâmica, uma simulação no domínio do tempo. Nas simulações envolvendo transformadores de tapes variáveis, foi possível verificar a existência o efeito reverso da ação de controle de tensão nas análises estática e dinâmica. Nas simulações utilizando o compensador estático de potência reativa houve divergência entre os resultados das duas análises.

\section{Palavras - chave}

Estabilidade de tensão; instabilidade de tensão; LTC; transformadores; simulação estática; simulação dinâmica; compensador estático de reativos. 


\section{Abstract}

Pontes, Bruno do Carmo; Prada, Ricardo Bernardo. Static and Dynamic Simulation for the Voltage Control by LTC and Static Voltage Compensator. Rio de Janeiro, 2008. 194p. Master Dissertation - Departamento de Engenharia Elétrica, Pontifícia Universidade Católica do Rio de Janeiro.

This work presents the observation and analysis, in steady state and dynamic performance, of the phenomenon already observed in real operation conditions of the Brazilian Electric System, which is the opposite relationship between the controlled value and the target value. In this case, even if the resources have margin to keep the voltage controlled, this is not useful. For example, the reduction in the turn ratio on load tap changer transformer, with the aim of increase in the controlled voltage, result in its reduction, until the tap changer limit is reached or the system is led to the collapse. To demonstrate the existence of this problem, steady state and dynamic performance simulations were done, and the voltage control effect by on load tap changer transformer and static var compensator, current situations in a power system. Several situations where is possible verify the operation region changing was demonstrated. For steady state analysis was used a load flow algorithm and, for the dynamic analysis, a time domain simulation. In the simulations with on load tap changer transformer, it was possible to verify the existence of the reverse effect of the voltage control action in the static and dynamic analysis. In the simulations using static var compensator, a divergence was found between the results in the two analyses.

\section{Key-Words}

Voltage stability, voltage instability, LTC, transformers, static simulation, dynamic simulation, static voltage compensator. 


\section{Sumário}

1 Introdução 26

1.1 O Problema da Estabilidade de Tensão 28

1.2 Casos de Instabilidade de Tensão no Sistema Brasileiro 29

1.3 Instabilidade de Tensão em 24/04/1997 às 18:15 h 30

1.4 Instabilidade de Tensão em 25/04/1997 às 18:17 h 31

1.5 Instabilidade de Tensão em 13/11/1997 às 9:25 h 32

1.6 Organização do Trabalho 33

2 Estabilidade de Tensão [6] 34

2.1 Introdução 34

2.2 Equações de Fluxo de Potência Ativa e Reativa Injetada na Barra de Carga 34

2.3 Curvas $\mathrm{P}, \mathrm{Q}$ e $\phi$ Constantes 36

2.4 O Limite de Estabilidade de Tensão (LET) 38

2.5 A Existência da Potência Transmitida "Maximum Maximorum" 42

2.6 O Porquê da Potência Transmitida Máxima para a Carga 46

2.7 Ponto de operação na parte superior da curva 48

2.8 Ponto de operação na parte inferior da curva 49

2.9 O Porquê da Introdução de um Capacitor Diminui a Tensão 50

2.9.1 Ponto de operação na parte superior da curva 52

2.9.2 Ponto de operação na parte inferior da curva 53

$2.10 \quad$ Análise dos resultados 53

$3 \quad$ LTC - Load Tap Change 54

3.1 Introdução 54

3.2 Modelagem dos LTCs para Análises de Regime Permanente [8] 54

3.2.1 LTCs com Variação de Tapes no Primário 54

3.2.2 LTCs com Variação de Tapes no Secundário 58

3.3 Modelagem de um LTC nos Programa de Fluxo de Carga [9] 61

3.4 Representação dos Controles e Cálculo dos Índices de Estabilidade de Tensão $[7,9] \quad 62$ 
3.4.1 Índice Tape do LTC x Tensão da Barra Controlada Localmente

62

3.4.2 Índice Tape do LTC x Tensão da Barra Controlada Remotamente $\quad 65$

3.5 Equações da Matriz Jacobiana 66

$\begin{array}{lll}3.6 & \text { Análise dos Resultados } & 67\end{array}$

4 CER - Compensador Estático de Potência Reativa 68

$4.1 \quad$ Introdução 68

4.2 Reatância e Susceptância do CER[9] 68

4.3 Modelagem do CER nos Programas de Fluxo de Potência 71

4.4 Equações de Controle para o Cálculo dos Índices 75

4.5 Estrutura da Matriz Jacobiana e Cálculo dos Índices 76

4.5.1 Índice Susceptância x Tensão Terminal (ou Remota) 76

4.5.1.1 Modelo de injeção de corrente - Controle de Tensão Local

$\begin{array}{ll}\left(\mathrm{V}_{\text {cont }}=\mathrm{V}_{\mathrm{t}}\right) & 77\end{array}$

4.5.1.2 Modelo de injeção de corrente - Controle de Tensão Remoto $\left(\mathrm{V}_{\text {cont }} \neq \mathrm{V}_{\mathrm{t}}\right) \quad 77$

4.5.1.3 Modelo de injeção de potência - Controle de Tensão Local $\left(\mathrm{V}_{\text {cont }}=\mathrm{V}_{\mathrm{t}}\right) \quad 78$

4.5.1.4 Modelo de injeção de potência - Controle de Tensão Remoto $\left(\mathrm{V}_{\text {cont }} \neq \mathrm{V}_{\mathrm{t}}\right) \quad 78$

4.5.2 Índice Ângulo de Disparo dos Tiristores $x$ Tensão Terminal (ou Remota) $\quad 79$

4.5.2.1 Modelo de injeção de corrente - Controle de Tensão Local $\left(\mathrm{V}_{\text {cont }}=\mathrm{V}_{\mathrm{t}}\right) \quad 80$

4.5.2.2 Modelo de injeção de corrente - Controle de Tensão Remoto $\left(\mathrm{V}_{\text {cont }} \neq \mathrm{V}_{\mathrm{t}}\right) \quad 80$

4.5.2.3 Modelo de injeção de potência - Controle de Tensão Local $\left(\mathrm{V}_{\text {cont }}=\mathrm{V}_{\mathrm{t}}\right) \quad 81$

4.5.2.4 Modelo de injeção de potência - Controle de Tensão Remoto $\left(\mathrm{V}_{\text {cont }} \neq \mathrm{Vt}_{\mathrm{Vt}}\right) \quad 81$

4.6 Exemplo Numérico [9] 83

4.6.1 Cálculo do Índice Susceptância x Tensão Terminal 85

4.6.2 Cálculo do Índice Ângulo de Disparo dos Tiristores x Tensão Terminal 86

4.6.3 Resultados de 4.6.1 e 4.6.2 88 
4.7 Análise dos Resultados

$5 \quad$ Análise do Efeito do Controle de Tensão com LTCs 92

5.1 Introdução 92

$5.2 \quad$ Modelagem do LTC 92

5.3 Modelagem dos Reguladores de Tensão 95

5.4 Modelagem da Carga 96

5.5 Carga "Potência Constante" 96

5.5.1 Testes no Sistema de 3 Barras com a Carga "Potência

Constante" $\quad 97$

5.5.1.1 Região Normal de Operação $\quad 97$

5.5.1.2 Região Anormal de Operação 102

5.6 Carga "Impedância Constante" 107

5.6.1 Testes do Sistema de 3 Barras com a Carga "Impedância

Constante" 108

$\begin{array}{lll}\text { 5.6.1.1 Região Normal de Operação } & 108\end{array}$

5.7 Carga "Corrente Constante" 113

5.8 Carga "ZIP" 114

5.8.1 Testes do Sistema de 3 Barras com a Carga "ZIP" 115

5.8.1.1 Região Normal de Operação 115

$\begin{array}{lll}\text { 5.8.1.2 Região Anormal de Operação } & 121\end{array}$

$\begin{array}{lll}5.9 & \text { Análise dos Resultados } & 127\end{array}$

6 Análise do Efeito do Controle de Tensão com LTCs em um

Sistema-Teste de 10 barras, em Regime Permanente e Dinâmico 129

$\begin{array}{lll}6.1 & 129\end{array}$

6.2 Controle de Tensão por LTC Automático 130

6.3 Análise em Regime Permanente 130

$\begin{array}{lll}\text { 6.3.1 } & \text { Região Normal de Operação } & 131\end{array}$

6.3.2 Região Anormal de Operação 132

6.4 Análise no Domínio do Tempo 134

$\begin{array}{lll}\text { 6.4.1 Região Normal de Operação } & 134\end{array}$

$\begin{array}{lll}6.4 .2 & \text { Região Anormal de Operação } & 137\end{array}$

6.5 Controle de Tensão por LTC Manual 140

6.5.1 Análise em Regime Permanente 140

6.5.1.1 Regiões Normal e Anormal de Operação 140

6.5.2 Análise no Domínio do Tempo 143 
6.5.2.1 Regiões Normal e Anormal de Operação 143

$\begin{array}{lll}6.6 & \text { Análise dos resultados } & 144\end{array}$

7 Análise do Efeito do Controle de Tensão com Compensadores

Estáticos de Reativos (CER) em um Sistema-Teste de 39 Barras, em Regime Permanente e Dinâmico 146

$\begin{array}{lll}7.1 & 146\end{array}$

7.2 Análise em Regime Permanente 151

7.2.1 Regiões Normal e Anormal de Operação 151

7.3 Análise no Domínio do Tempo 154

7.3.1 Região Normal de Operação 154

$\begin{array}{lll}\text { 7.3.2 } & \text { Região Anormal de Operação } & 161\end{array}$

$\begin{array}{lll}7.4 & \text { Análise dos resultados } & 174\end{array}$

$8 \quad$ Conclusão e Sugestões para Trabalhos Futuros 175

$\begin{array}{lll}8.1 & \text { Sugestões para trabalhos futuros } & 177\end{array}$

9 Referências Bibliográficas 178

10 Apêndice 180

10.1 Arquivo base do ANATEM das simulações do Capítulo $5 \quad 181$

10.2 Complemento de 10.1 para a simulação da Figura 3.26 e

Figura $3.27 \quad 181$

10.3 Complemento de 10.1 para a simulação da Figura 3.28,

Figura 3.29, Figura 3.30 e Figura $3.31 \quad 181$

10.4 Complemento de 10.2 para a simulação da Figura 3.33 e

Figura $3.34 \quad 182$

10.5 Complemento de 10.3 para a simulação da Figura 3.35,

Figura 3.36, Figura 3.37 e Figura 3.38

10.6 Complemento de 10.1 para a simulação da Figura 3.41 e

Figura $3.42 \quad 182$

10.7 Complemento de 10.6 para a simulação da Figura 3.43,

Figura 3.44, Figura 3.45 e Figura $3.46 \quad 183$

10.8 Complemento de 10.1 para a simulação da Figura 3.50 e

Figura $3.51 \quad 183$

10.9 Complemento de 10.8 para a simulação da Figura 3.52

,Figura 3.53, Figura 3.54 e Figura 3.55 
10.10

Complemento de 10.8 para a simulação da Figura 3.56 e

Figura 3.57

184

10.11

Complemento de 10.8 para a simulação da Figura 3.59 e

Figura 3.60

185

10.12 Complemento de 10.9 para a simulação da Figura 3.61,

Figura 3.62, Figura 3.63 e Figura 3.64

185

10.13 Complemento de 10.10 para a simulação da Figura 3.65 e

Figura 3.66

185

10.14

Arquivo base do ANATEM das simulações da Figura 6.4 a

Figura 6.9

186

10.15

Complemento de 10.14 para a simulação da Figura 6.4

186

10.16

Complemento de 10.15 para a simulação da Figura 6.5

186

10.17

Complemento de 10.15 para a simulação da Figura 6.6

186

10.18

Complemento de 10.15 para a simulação da Figura 6.7

187

10.19

Complemento de 10.16 para a simulação da Figura 6.8

187

10.20

Complemento de 10.17 para a simulação da Figura 6.9

187

10.21

Arquivo completo do ANATEM para a simulação da Figura

6.11 e Figura 6.12

187

10.22

Arquivo base do ANATEM das simulações do Capítulo 7

189

10.23 Complemento de 10.22 para a simulação da Figura 7.5 a

Figura 7.8 e Figura 7.17 a Figura 7.20 (alteração apenas do caso base de regime permanente)

10.24 Complemento de 10.22 para a simulação da Figura 7.9 a

Figura 7.12 e Figura 7.21 a Figura 7.24 (alteração apenas do caso base de regime permanente)

10.25 Complemento de 10.22 para a simulação da Figura 7.13 a

Figura 7.16 e Figura 7.25 a Figura 7.28 (alteração apenas do caso base de regime permanente)

10.26 Complemento de 10.22 para a simulação da Figura 7.29 e

Figura 7.30

10.27

Complemento de 10.22 para a simulação da Figura 7.31 e

Figura 7.32

10.28

Complemento de 10.22 para a simulação da Figura 7.33 a

Figura 7.36 


\section{Lista de Tabelas}

Tabela 2.1 - Três Possibilidades de Solução para a Tensão na Carga com Mesmo Fator de Potência

Tabela 2.2 - Variações de Tensão, Corrente e Potência na Barra de Carga

Tabela 2.3 - Pontos de Operação para Avaliar o Aumento ou Decréscimo da Tensão com a Introdução de um Capacitor

Tabela 3.1 - Ponto de operação com a barra controlada na região anormal de operação

Tabela 3.2 - Ponto de operação com a barra controlada na região normal de operação

Tabela 4.1 - Dados de Linha do Sistema de 5 Barras

Tabela 4.2 - Ponto de operação inicial na região normal de operação

Tabela 4.3 - Efeito do aumento de $V_{\text {esp }}$ no ponto de operação da Tabela

4.2

Tabela 4.4 - Ponto de operação inicial na região anormal de operação

Tabela 4.5 - Efeito do Aumento de $V_{\text {esp }}$ no ponto de Operação da Tabela 4.4

Tabela 5.1 - Ponto de operação inicial

Tabela 5.2 - Resultado da variação do tape para variações na tensão controlada, carga "potência constante"

Tabela 5.3 - Ponto de operação inicial

Tabela 5.4 - Resultado da variação do tape para variações na tensão controlada, carga "potência constante"

Tabela 5.5 - Ponto de operação inicial

Tabela 5.6 - Resultado da variação do tape para variações da tensão controlada, carga "impedância constante"

Tabela 5.7 - Ponto de operação inicial

Tabela 5.8 - Resultado da variação do tape em virtude da variação na tensão controlada, carga "ZIP" 
Tabela 5.10 - Resultado da variação do tape em virtude da variação da tensão controlada, carga "ZIP"

Tabela 5.11 - Comparação dos pontos de operação das simulações estática e dinâmica

Tabela 6.1 - Dados dos circuitos CA do sistema-teste de 10 barras

Tabela 6.2 - Dados dos transformadores do sistema-teste de 10 barras

Tabela 6.3 - Ponto de operação inicial

Tabela 6.4 - Posição dos tapes no ponto de operação inicial

Tabela 6.5 - Resultado da variação da tensão controlada (barra 11) e

tape do transformador entre as barras 10 e 11

Tabela 6.6 - Ponto de operação inicial

Tabela 6.7 - Posição dos tapes no ponto de operação inicial

Tabela 6.8 - Resultado da variação do módulo da tensão controlada (barra 11), da tensão na barra 10 e do tape do transformador entre as barras 10 e 11

Tabela 6.9 - Pontos iniciais $(0 \mathrm{~s})$ e finais (20 s) da simulação no domínio do tempo

Tabela 6.10 - Pontos iniciais (0 s) e finais $(20 \mathrm{~s})$ da simulação no domínio do tempo, com limitação de atuação do LTC

Tabela 6.11 - Pontos iniciais (0 s) e finais $(20 \mathrm{~s})$ da simulação no domínio do tempo, com limitação de atuação do LTC

Tabela 6.12 - Ponto de operação inicial

Tabela 6.13 - Posição dos tapes no ponto de operação inicial

Tabela 6.14 - Resultado da variação do tape do transformador entre as barras 10 e 11 e dos módulos das tensões nas barras 10 e 11

Tabela 6.15 - Módulo das tensões das barras 10 e 11 e tape do transformador entre estas duas barras, retirados da simulação no domínio do tempo

Tabela 7.1 - Dados dos circuitos CA do sistema-teste de 39 barras 148

Tabela 7.2 - Dados dos transformadores do sistema-teste de 39 barras 149

Tabela 7.3 - Ponto de operação inicial

Tabela 7.4 - Resultado da variação da potência reativa gerada pelo CER, da tensão controlada (barra 16) e da susceptância do compensador

Tabela 7.5 - Pontos iniciais (0 s) e finais (10 s) da simulação no domínio do tempo, com regulador do CER built-in

Tabela 7.6 - Pontos iniciais ( $0 \mathrm{~s})$ e finais (10 s) da simulação no domínio do tempo, com regulador do CER customizado 
Tabela 7.7 - Pontos iniciais ( $0 \mathrm{~s}$ ) e em $\mathrm{t}=10 \mathrm{~s}$ da simulação no domínio do tempo, com regulador do CER customizado e sem regulador de tensão nos geradores

Tabela 7.8 - Pontos iniciais (0 s) e finais (10 s) da simulação no domínio do tempo, com regulador do CER built-in

Tabela 7.9 - Pontos iniciais (0 s) e finais (10 s) da simulação no domínio do tempo, com regulador do CER customizado

Tabela 7.10 - Pontos iniciais (0 s) e em $t=10 \mathrm{~s}$ da simulação no domínio do tempo, com regulador do CER customizado e sem regulador de tensão nos geradores

Tabela 7.11 - Pontos de operação em 0s (4), 4+ s (5), 8+ s (6) 12+s (7), $16+s$ (8) da simulação no domínio do tempo, com regulador do CER builtin e reguladores de tensão dos geradores como barra infinita

Tabela 7.12 - Pontos de operação em 0s (4), 4+ s (5), 8+ s (6) 12+s (7), 16 + s (8) da simulação no domínio do tempo, com regulador do CER customizados e reguladores de tensão dos geradores como barra infinita 173 Tabela 7.13 - Pontos de operação em 0s (4), 4+ s (5), 8+ s (6) 12+s (7), 16 + s (8) da simulação no domínio do tempo, com regulador do CER customizado e sem reguladores de tensão nos geradores 


\section{Lista de Figuras}

Figura 1.1 - Sistema Interligado Nacional horizonte 2009 com a previsão de interligação do sistema Acre-Rondônia [3]

Figura 1.2 - Curva de carga típica do SIN em dias úteis [1]

Figura 2.1 - Sistema Série de Duas Barras

Figura 2.2 - Três Possibilidades de Solução para a Tensão na Carga com Mesmo fator de Potência

Figura 2.3 - Curva para Fator de Potência Constante na Barra de Carga no Plano SV

Figura 2.4 - Circuito com as Impedâncias da Transmissão e da Carga 39

Figura 2.5 - Limite de Estabilidade de Tensão no Plano SV

Figura 2.6 - Lugar Geométrico da Tensão na Carga para Todos os Possíveis Diferentes Níveis de Potência Ativa Constante e Para Alguns Níveis de Potência Reativa Constante

Figura 2.7 - Aumento e Diminuição da Tensão Respectivamente na Região Superior e Inferior da Curva com a Introdução de um Capacitor

Figura 2.8 - Potência Ativa Consumida na Carga com Fator de Potência Constante

Figura 2.9 - Circuito sem Capacitor 50

Figura 2.10 - Circuito com Capacitor 50

Figura 3.1 - Modelagem $\pi$ clássica de linhas de transmissão 55

Figura 3.2 - Modelagem de LTCs com variação de tapes no primário 55

Figura 3.3 - Circuito $\pi$ equivalente de um LTC

Figura 3.4 - Circuito $\pi$ equivalente de um LTC com parâmetros expressos em função da admitância e relação de transformação

Figura 3.5 - Circuito $\pi$ equivalente de um LTC com parâmetros expressos em função da impedância e relação de transformação

Figura 3.6 - Modelagem de LTCs com variação de tapes no secundário

Figura 3.7 - Circuito $\pi$ equivalente de um LTC com parâmetros expressos em função da admitância e relação de transformação 
Figura 3.8 - Circuito $\pi$ equivalente de um LTC com parâmetros expressos em função da impedância e relação de transformação

Figura 3.9 - Sistema de 3 Barras com LTC 62

Figura 4.1 - Estrutura do CER 69

Figura 4.2 - Reatância equivalente do CER em função do ângulo de disparo dos tiristores $\left(X_{C}=15 \Omega\right.$ e $\left.X_{L}=2,56 \Omega\right)$

Figura 4.3 - Susceptância equivalente do CER em função do ângulo de disparo dos tiristores $\left(\mathrm{X}_{\mathrm{C}}=15 \Omega\right.$ e $\left.\mathrm{X}_{\mathrm{L}}=2,56 \Omega\right)$

Figura 4.4 - Característica VI em Regime Permanente do CER [11] 71

Figura 4.5 - Característica VQ em Regime Permanente do CER [11] 72

Figura 4.6 - Efeito esperado da variação de a sobre $V_{\text {cont }}$

Figura 4.7 - Efeito oposto ao esperado da variação de $\alpha$ sobre $V_{\text {cont }}$

Figura 4.8 - Sistema de 5 Barras com CER 83

Figura 4.9 - Susceptâncias do FC, TCR em função do ângulo de disparo dos tiristores

Figura 4.10 - Susceptância do CER em função do ângulo de disparo dos tiristores

Figura 5.1 - Diagrama unifilar do sistema-teste de 3 barras

Figura 5.2 - Diagrama de blocos do modelo de LTC automático MD01 do ANATEM

Figura 5.3 - Diagrama de blocos do regulador de tensão MD20 do ANATEM

Figura 5.4 - Diagrama de blocos do regulador de tensão customizado

Figura 5.5 - Curvas PV de um sistema com carga potência constante para diferentes valores de tape

Figura 5.6 - Resultado da variação da tensão da barra $1 X$ tape, carga "potência constante"

Figura 5.7 - Resultado da variação da tensão da barra 0, carga "potência constante", no domínio do tempo, regulador de tensão MD20 do ANATEM Figura 5.8 - Resultado da variação da tensão da barra 1 e do tape, carga "potência constante", no domínio do tempo , regulador de tensão MD20 do ANATEM

Figura 5.9 - Resultado da variação da tensão das 3 barras, carga "potência constante", no domínio do tempo , regulador de tensão barra infinita

Figura 5.10 - Resultado da variação do tape, carga "potência constante", no domínio do tempo, regulador de tensão barra infinita 
Figura 5.11 - Resultado da variação das potências geradas, carga "potência constante", no domínio do tempo , regulador de tensão barra infinita

Figura 5.12 - Resultado da variação das potências consumidas, carga "potência constante", no domínio do tempo , regulador de tensão barra infinita

Figura 5.13 - Resultado da variação da tensão da barra $1 \mathrm{X}$ tape, carga "potência constante"

Figura 5.14 - Resultado da variação da tensão da barra 0 , carga "potência constante", no domínio do tempo, regulador de tensão MD20 do ANATEM

Figura 5.15 - Resultado da variação da tensão da barra 1 e do tape, carga "potência constante", no domínio do tempo , regulador de tensão MD20 do ANATEM

Figura 5.16 - Resultado da variação da tensão das 3 barras, carga "potência constante", no domínio do tempo , regulador de tensão barra infinita

Figura 5.17 - Resultado da variação do tape, carga "potência constante", no domínio do tempo, regulador de tensão barra infinita

Figura 5.18 - Resultado da variação das potências geradas, carga "potência constante", no domínio do tempo , regulador de tensão barra infinita

Figura 5.19 - Resultado da variação das potências consumidas, carga "potência constante", no domínio do tempo , regulador de tensão barra infinita

Figura 5.20 - Curvas PV para diferentes valores de tape e curvas de carga tipo "impedância constante" e o efeito da troca de tape em pontos de operação na parte superior e inferior da curva PV

Figura 5.21 - Resultado da variação da tensão da barra $1 \mathrm{X}$ tape, carga "impedância constante"

Figura 5.22 - Resultado da variação da tensão da barra 0 , carga "impedância constante", no domínio do tempo, regulador de tensão MD20 do ANATEM

Figura 5.23 - Resultado da variação da tensão da barra 1 e do tape, carga "impedância constante", no domínio do tempo , regulador de tensão MD20 do ANATEM 
Figura 5.24 - Resultado da variação da tensão das 3 barras, carga "impedância constante", no domínio do tempo , regulador de tensão barra infinita

Figura 5.25 - Resultado da variação do tape, carga "impedância constante", no domínio do tempo, regulador de tensão barra infinita

Figura 5.26 - Resultado da variação das potências geradas, carga "impedância constante", no domínio do tempo , regulador de tensão barra infinita

Figura 5.27 - Resultado da variação das potências consumidas, carga "impedância constante", no domínio do tempo , regulador de tensão barra infinita

Figura 5.28 - Curvas PV para dois valores de tape, a curva de carga tipo "corrente constante", e o efeito da troca de tape

Figura 5.29 - Curvas PV para dois valores de tape, curva de carga tipo "ZIP" e o efeito da troca de tape em pontos de operação na parte superior e inferior da curva PV

Figura 5.30 - Resultado da variação da tensão da barra $1 \mathrm{X}$ tape, carga "ZIP"

Figura 5.31 - Resultado da variação da tensão da barra 0, carga "ZIP", no domínio do tempo, regulador de tensão MD20 do ANATEM

Figura 5.32 - Resultado da variação da tensão da barra 1 e do tape, carga "ZIP", no domínio do tempo, regulador de tensão MD20 do ANATEM

Figura 5.33 - Resultado da variação da tensão das 3 barras, carga "ZIP", no domínio do tempo, regulador de tensão barra infinita

Figura 5.34 - Resultado da variação do tape, carga "ZIP", no domínio do tempo, regulador de tensão barra infinita

Figura 5.35 - Resultado da variação das potências geradas, carga "ZIP", no domínio do tempo, regulador de tensão barra infinita

Figura 5.36 - Resultado da variação das potências consumidas, carga "ZIP", no domínio do tempo, regulador de tensão barra infinita

Figura 5.37 - Resultado da variação da tensão da barra de geração e de carga, carga "ZIP", no domínio do tempo, regulador de tensão customizado

Figura 5.38 - Resultado da variação do tensão da barra terminal do transformador e do tape, carga "ZIP", no domínio do tempo, regulador de tensão barra customizado 
Figura 5.39 - Resultado da variação da tensão da barra $1 \mathrm{X}$ tape, carga "ZIP"

Figura 5.40 - Resultado da variação da tensão da barra 0, carga "ZIP", no domínio do tempo, regulador de tensão MD20 do ANATEM

Figura 5.41 - Resultado da variação da tensão da barra 1 e do tape, carga "ZIP", no domínio do tempo, regulador de tensão MD20 do ANATEM

Figura 5.42 - Resultado da variação da tensão das 3 barras, carga "ZIP", no domínio do tempo, regulador de tensão barra infinita

Figura 5.43 - Resultado da variação do tape, carga "ZIP", no domínio do tempo, regulador de tensão barra infinita

Figura 5.44 - Resultado da variação das potências geradas, carga "ZIP", no domínio do tempo, regulador de tensão barra infinita

Figura 5.45 - Resultado da variação das potências consumidas, carga "ZIP", no domínio do tempo, regulador de tensão barra infinita

Figura 5.46 - Resultado da variação da tensão da barra de geração e de carga, carga "ZIP", no domínio do tempo, regulador de tensão customizado

Figura 5.47 - Resultado da variação do tensão da barra terminal do transformador e do tape, carga "ZIP", no domínio do tempo, regulador de tensão barra customizado

Figura 6.1 - Diagrama unifilar do sistema-teste de 10 barras

Figura 6.2 - Resultado da variação da tensão da barra $11 \mathrm{X}$ tape do transformador entre as barras 10 e 11

Figura 6.3 - Resultado da variação da tensão da barra $11 \times$ tape do transformador entre as barras 10 e 11

Figura 6.4 - Resultado da variação da tensão das barras 10, do tape contínuo e ilimitado e da tensão da barra 11, no domínio do tempo

Figura 6.5 - Resultado da variação da tensão das barras 10, do tape contínuo e limitado e da tensão da barra 11, no domínio do tempo

Figura 6.6 - Resultado da variação da tensão das barras 10, do tape discreto e limitado e da tensão da barra 11, no domínio do tempo

Figura 6.7 - Resultado da variação da tensão da barra 11, do tape contínuo e iilimitado e da tensão da barra 10, no domínio do tempo

Figura 6.8 - Resultado da variação da tensão da barra 11, do tape contínuo e limitado e da tensão da barra 10, no domínio do tempo 
Figura 6.9 - Resultado da variação da tensão das barras 10, do tape discreto e limitado e da tensão da barra 11, no domínio do tempo

Figura 6.10 - Resultado da variação da tensão da barra $11 \mathrm{X}$ tape do transformador entre as barras 10 e 11

Figura 6.11 - Resultado da variação do módulo da tensão das barras 10 e 11, para incrementos sucessivos no tape, no domínio do tempo

Figura 6.12 - Alteração imposta ao tape do transformador entre as barras 10 e 11, no domínio do tempo

Figura 7.1 - Diagrama unifilar do sistema-teste de IEEE-39 barras New England

Figura 7.2 - Modelo built-in para simulação dinâmica do CER

Figura 7.3 - Modelo customizado para simulação dinâmica do CER

Figura 7.4 - Resultado da variação da tensão da barra 16 x susceptância do CER

Figura 7.5 - Resultado da variação da tensão dos geradores ligados às barras 30 a 34, no domínio do tempo, simulação com regulador do CER built-in e reguladores de tensão dos geradores como barra infinita

Figura 7.6 - Resultado da variação da tensão dos geradores ligados às barras 35 a 39, no domínio do tempo, simulação com regulador do CER built-in e reguladores de tensão dos geradores como barra infinita

Figura 7.7 - Resultado da variação da tensão da barra 16, no domínio do tempo, simulação com regulador do CER built-in e reguladores de tensão dos geradores como barra infinita

Figura 7.8 - Resultado da variação da susceptância do CER, no domínio do tempo, simulação com regulador do CER built-in e reguladores de tensão dos geradores como barra infinita

Figura 7.9 - Resultado da variação da tensão dos geradores ligados às barras 30 a 34, no domínio do tempo, simulação com regulador do CER customizado e reguladores de tensão dos geradores como barra infinita

Figura 7.10 - Resultado da variação da tensão dos geradores ligados às barras 35 a 39, no domínio do tempo, simulação com regulador do CER customizado e reguladores de tensão dos geradores como barra infinita

Figura 7.11 - Resultado da variação da tensão da barra 16, no domínio do tempo, simulação com regulador do CER customizado e reguladores de tensão dos geradores como barra infinita 
Figura 7.12 - Resultado da variação da susceptância do CER, no domínio do tempo, simulação com regulador do CER customizado e reguladores de tensão dos geradores como barra infinita

Figura 7.13 - Resultado da variação da tensão dos geradores ligados às barras 30 a 34, no domínio do tempo, simulação com regulador do CER customizado e sem reguladores de tensão dos geradores

Figura 7.14 - Resultado da variação da tensão dos geradores ligados às barras 35 a 39, no domínio do tempo, simulação com regulador do CER customizado e sem reguladores de tensão nos geradores

Figura 7.15 - Resultado da variação da tensão da barra 16, no domínio do tempo, simulação com regulador do CER customizado e sem reguladores de tensão nos geradores

Figura 7.16 - Resultado da variação da susceptância do CER, no domínio do tempo, simulação com regulador do CER customizado e sem reguladores de tensão nos geradores

Figura 7.17 - Resultado da variação da tensão dos geradores ligados às barras 30 a 34, no domínio do tempo, simulação com regulador do CER built-in e reguladores de tensão dos geradores como barra infinita

Figura 7.18 - Resultado da variação da tensão dos geradores ligados às barras 35 a 39, no domínio do tempo, simulação com regulador do CER built-in e reguladores de tensão dos geradores como barra infinita

Figura 7.19 - Resultado da variação da tensão da barra 16, no domínio do tempo, simulação com regulador do CER built-in e reguladores de tensão dos geradores como barra infinita

Figura 7.20 - Resultado da variação da susceptância do CER, no domínio do tempo, simulação com regulador do CER built-in e reguladores de tensão dos geradores como barra infinita

Figura 7.21 - Resultado da variação da tensão dos geradores ligados às barras 30 a 34, no domínio do tempo, simulação com regulador do CER customizado e reguladores de tensão dos geradores como barra infinita

Figura 7.22 - Resultado da variação da tensão dos geradores ligados às barras 35 a 39, no domínio do tempo, simulação com regulador do CER customizado e reguladores de tensão dos geradores como barra infinita

Figura 7.23 - Resultado da variação da tensão da barra 16, no domínio do tempo, simulação com regulador do CER customizado e reguladores de tensão dos geradores como barra infinita 
Figura 7.24 - Resultado da variação da susceptância do CER, no domínio do tempo, simulação com regulador do CER customizado e reguladores de tensão dos geradores como barra infinita

Figura 7.25 - Resultado da variação da tensão dos geradores ligados às barras 30 a 34, no domínio do tempo, simulação com regulador do CER customizado e sem reguladores de tensão dos geradores

Figura 7.26 - Resultado da variação da tensão dos geradores ligados às barras 35 a 39, no domínio do tempo, simulação com regulador do CER customizado e sem reguladores de tensão nos geradores

Figura 7.27 - Resultado da variação da tensão da barra 16, no domínio do tempo, simulação com regulador do CER customizado e sem reguladores de tensão nos geradores

Figura 7.28 - Resultado da variação da susceptância do CER, no domínio do tempo, simulação com regulador do CER customizado e sem reguladores de tensão nos geradores

Figura 7.29 - Resultado da variação da tensão controlada pelo CER, no domínio do tempo, simulação com regulador do CER built-in e reguladores de tensão dos geradores como barra infinita

Figura 7.30 - Resultado da variação da susceptância do CER, no domínio do tempo, simulação com regulador do CER built-in e reguladores de tensão dos geradores como barra infinita

Figura 7.31 - Resultado da variação da tensão controlada pelo CER, no domínio do tempo, simulação com regulador do CER customizados e reguladores de tensão dos geradores como barra infinita

Figura 7.32 - Resultado da variação da susceptância do CER, no domínio do tempo, simulação com regulador do CER customizados e reguladores de tensão dos geradores como barra infinita

Figura 7.33 - Resultado da variação da tensão dos geradores, no domínio do tempo, simulação com regulador do CER customizado e sem reguladores de tensão nos geradores

Figura 7.34 - Resultado da variação da tensão dos geraodres, no domínio do tempo, simulação com regulador do CER customizado e sem reguladores de tensão nos geradores

Figura 7.35 - Resultado da variação da tensão controlada pelo CER, no domínio do tempo, simulação com regulador do CER customizado e sem reguladores de tensão nos geradores 
Figura 7.36 - Resultado da variação da susceptância do CER, no domínio do tempo, simulação com regulador do CER customizado e sem reguladores de tensão nos geradores 


\section{Siglas e Acrônimos}

\begin{tabular}{|c|c|}
\hline ANEEL & Agência Nacional de Energia Elétrica \\
\hline $\mathrm{CA}$ & Corrente Alternada \\
\hline CAG & Controle Automático de Geração \\
\hline CCAT & Corrente Contínua em Alta Tensão \\
\hline CDU & Controlador Definido pelo Usuário \\
\hline CE/CER & Compensador Estático de Reativos \\
\hline Cemig & Companhia Energética de Minas Gerais \\
\hline CNOS & Centro Nacional de Operação do Sistema \\
\hline CS & Compensador Síncrono \\
\hline ECE & Esquema de Controle de Emergência \\
\hline Eletrobrás & Centrais Elétricas Brasileiras S.A. \\
\hline Eletropaulo & Eletropaulo - Eletricidade de São Paulo S.A. \\
\hline ERAC & Esquema Regional de Alívio de Carga \\
\hline Escelsa & Espírito Santo Centrais Elétricas S.A. \\
\hline FC & Fixed Capacitor \\
\hline LET & Limite de Estabilidade de Tensão \\
\hline LTC & Load Tap Change \\
\hline ONS & Operador Nacional do Sistema \\
\hline OXL & Over Excitation Limiter \\
\hline RESEB & Reestruturação do Sistema Elétrico Brasileiro \\
\hline SE & Subestação \\
\hline SEP & Sistema Elétrico de Potência \\
\hline SIN & Sistema Interligado Nacional \\
\hline SVC & Static var Compensator \\
\hline TCR & Thyristor Controlled Reactor \\
\hline UHE & Usina Hidroelétrica \\
\hline
\end{tabular}

